\title{
A Flexible Adjustment and Control System for Hydraulic Machines
}

\author{
Daniel Banyai ${ }^{1}$, Lucian Marcu ${ }^{1}$ \\ ${ }^{1}$ Technical University of Cluj-Napoca, Department of Mechanical Engineering, B-dul Muncii, nr. 103-105, Cluj-Napoca, \\ Romania
}

Correspondence to: daniel.banyai@termo.utcluj.ro

\begin{abstract}
Due to the advantages of hydraulic systems with variable displacement, it was necessary to design a control system that can adjust the pressure, flow, power or a combination of these features, that can be easily integrated into the pump body without changing its mechanical construction. The objective of this work was to study the dynamic behavior of this electro-hydraulic control system. To achieve these objectives, first the adjusting system was analyzed by numerical simulations, and then a stand was constructed for testing the performance of these adjustable pumps. It was shown that this control system is superior to existing systems.
\end{abstract}

Keywords: adjustable pump, control system, dynamic behavior.

\section{Introduction}

This paper presents a study of high power drives, namely hydraulic systems, pursuing a high degree of automation, minimum power consumption, adaptability to a large range of industrial applications and perturbations. The systems should be flexible.

Essential trends in the construction of presentday non-hydraulic machines are toward flexibility and automation. The aim is to increase the level of intelligence of the machines and their adaptation to possible disturbances. [2]

Variable displacement pumps allow easy control of system parameters (pressure, flow, power, or combinations of these parameters). Their technical characteristics make them the best option for most applications, from machine tools to mobile devices. [2]

Companies with a tradition in manufacturing pumps and motors with axial piston and variable displacement (Rexroth, Bosch, Vickers, Parker) have been producing this type of machinery for highly automated systems since the 1980 , but there is little data in the literature on constructive solutions.

The biggest producers of hydraulic machines are opting for mechano-hydraulic control structures that can be used in circuits for regulating pressure, flow and power independently of each other, with each control parameter requiring a different type of constructive control structure [7].

\section{System description}

The purpose of this paper is to report on optimizations of the adjustment structure of hydraulic pa- rameters, their control in the system to which belong, and tests on the assembly pump-adjustment system.

Figure 1 presents a schematic diagram for an automatic control system proposed for implementation in a research program $[1,2]$. The system contains the following components: 1 - a variable displacement pump with axial pistons; 2 - a linear hydraulic motor for changing the angular position of the piston block holder, in order to modify the flow of the pump; 3 - a proportional directional valve that controls the position of the linear motor; 4 - pressure sensors; 5 - a diaphragm for measuring the flow rate of the pump; 6 - electronic circuits that calculate the pressure drop on the diaphragm, then determine the flow, and then the hydraulic power generated by the pump is obtained via a signal from a pressure sensor and the signal that represents the flow; 7 - an electronic comparator, designed to find the error between the programmed value and the actual value of the adjusted parameter (pressure, flow, power); 8 - an electronic controller, used to compensate the errors and give the command signal for the proportional valve; 9 - switches whose state determines the control structure; 10 - a fixed displacement pump, which provides the necessary flow for positioning the hydraulic motor. This flow can be taken from the flow of the adjustable pump, and in this case the auxiliary pump is no longer required; 11 - a relief valve, which protects the system from exceeding the permissible pressure in the hydraulic components. Thus, without a change in pump construction, this can be integrated into any control circuit for adjustable hydraulic machines, by simply actuating an electrical switch. 


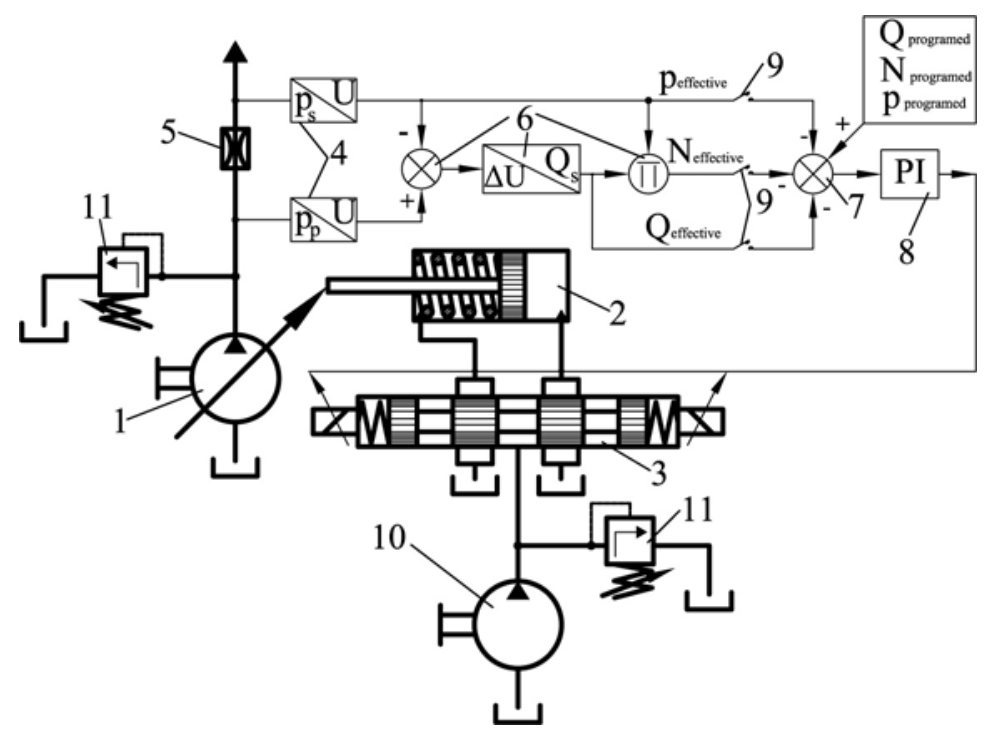

Figure 1: Electro-hydraulic control system for variable displacement machines

The mathematical model of the control system studied in this paper is based on differential equations that take into account nonlinear influences such as pressure and leakage flow dependence, saturation of the flow, limiting the pressure, etc., in order to minimize the deviations between the behavior of the real system and the modeled system.

The following differential equations are used to model the system [6]:

- equations of state (isothermal transformation) for discrete volumes; (a), (b);

- equation of continuity; (c), (d);

- equations describing the behavior of an ideal proportional electromagnet; (e);

- equations describing the behavior of an ideal controller; (f);

- mechanical equilibrium equations; $(\mathrm{g})$.

The general form of these equations was taken from the specialized literature $[3,6]$, and they were adapted to the model investigated in the paper, resulting in the system of equations described in (1), where the notations have the following meanings:

$\dot{p}_{A}$ - temporal derivative of the pressure function, $p_{A}$, in the large chamber of the linear hydraulic motor; $\dot{p}_{B}$ - temporal derivative of the pressure function, $p_{B}$, in the small chamber of the linear hydraulic motor; $p_{A}$ - pressure in the large chamber of the linear hydraulic motor; $p_{B}$ - pressure in the small chamber of the linear hydraulic motor; $p_{T}-$ tank pressure, $\left(p_{T}=0\right) ; p_{c}$ - pressure between the auxiliary pump and the control valve; $p_{s}-$ load pres- sure; $E_{U}$ - elasticity modulus; $V_{A}$ - volume of oil under pressure $p_{A} ; V_{B}$ - volume of oil under pressure $p_{B} ; V_{T}-($ dead) volume in the supply circuit of the linear motor, (volume of connecting pipes); $Q_{A}$ - flow rate that enters or is discharged from the large chamber of the positioning hydraulic motor; $Q_{B}$ - flow rate that enters or is discharged from the small chamber of the positioning hydraulic motor; $A$ - piston area (rodless); $x_{m}$ - linear position of the hydraulic motor; $c_{L G}$ - leakage flow coefficient dependent on speed; $c_{L P}$ - leakage flow coefficient dependent on pressure; $\alpha$ - piston surface ratio; $\alpha_{Q}-$ flow rate coefficient; $d_{v}$ - proportional valve diameter; $x_{v}$ - linear position of the proportional valve; $\rho$ - oil density; $T_{v}$ - time constant for the control valve; $K_{v}$ - gain of the control valve; $U$ - voltage generated by the PID controller; $U_{\text {ref }}$ - command voltage (command value for pressure, flow or power); $U_{r}$ - voltage generated by the sensors; $K_{p}$ - proportional gain, a tuning parameter of the PID controller; $T_{d}$ - derivative gain, a tuning parameter of the PID controller; $T_{i}$ - integral gain, a tuning parameter of the PID controller; $m_{p}$ - linear motor piston mass; $F_{a m}$ - preload force of the spring in the linear motor; $k_{m}-$ spring stiffness; $c_{3} ; c_{4}-$ viscous damping coefficient; $m$ - piston and rod of the reduced mass of the variable pump; $\omega-$ angular velocity of the piston holder of the pump; $R$ - placement radius of the pump's pistons; $a$ - tilting radius of the variable pump; $d$ - diameter of the pump's pistons.

$$
\begin{aligned}
& \dot{p}_{A}=\frac{E_{U}}{V_{A}+V_{T}} \cdot\left[Q_{A}-A \cdot \dot{x}_{m}-c_{L G} \cdot \dot{x}_{m}+c_{L P} \cdot\left(p_{A}-p_{B}\right)\right] \\
& \dot{p}_{B}=\frac{E_{U}}{V_{B}+V_{T}} \cdot\left[-Q_{B}+\alpha \cdot A \cdot \dot{x}_{m}-c_{L G} \cdot \dot{x}_{m}+c_{L P} \cdot\left(p_{A}-p_{B} t\right)\right]
\end{aligned}
$$




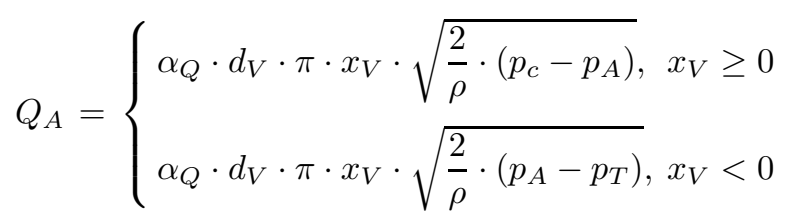

$$
\begin{aligned}
& Q_{B}=\left\{\begin{array}{l}
\alpha_{Q} \cdot d_{V} \cdot \pi \cdot x_{V} \cdot \sqrt{\frac{2}{\rho} \cdot\left(p_{B}-p_{T}\right)}, x_{V} \geq 0 \\
\alpha_{Q} \cdot d_{V} \cdot \pi \cdot x_{V} \cdot \sqrt{\frac{2}{\rho} \cdot\left(p_{c}-p_{B}\right)}, \quad x_{V}<0
\end{array}\right. \\
& T_{v} \cdot \dot{x}_{v}+x_{v}=K_{v} \cdot U \\
& U=K_{p} \cdot\left(U_{r e f}-U_{r}\right)+T_{d} \cdot\left(\dot{U}_{r e f}-\dot{U}_{r}\right)+\frac{1}{T_{i}} \int\left(U_{r e f}-U_{r}\right) \cdot \mathrm{d} t \\
& m_{p} \cdot \ddot{x}_{m}=A \cdot p_{A}-\alpha \cdot A \cdot p_{B}+F_{a m}+k_{m} \cdot x_{m}-\left(c_{3}+c_{4}\right) \dot{x}_{m}+\frac{m \cdot \omega^{2} \cdot R^{2}}{a^{2}} \cdot x_{m}-\frac{\pi \cdot d^{2} \cdot R}{4 a} \cdot p_{s}
\end{aligned}
$$

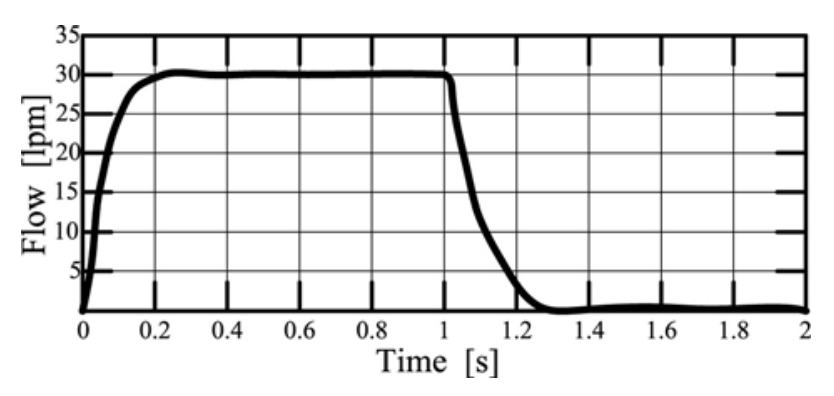

Figure 2: Pressure control

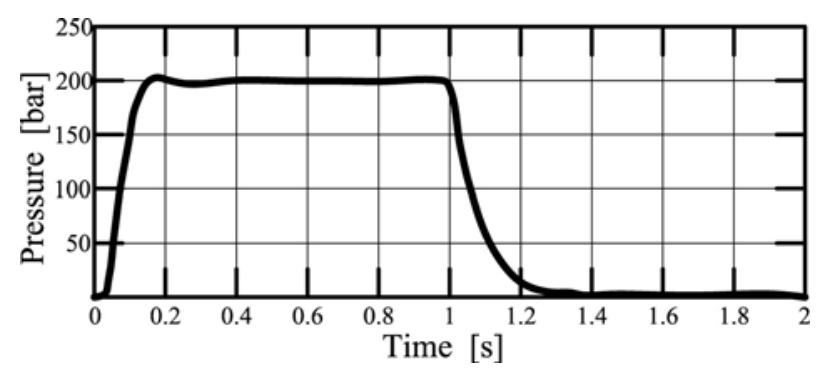

Figure 3: Flow control

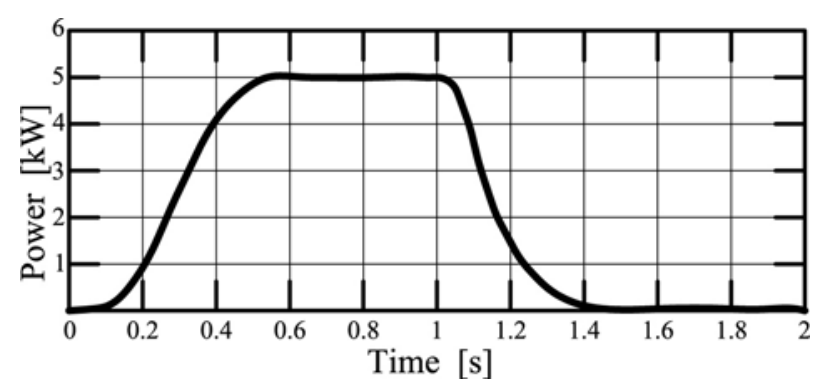

Figure 4: Power control

\section{$3 \quad$ Numerical research}

A study of the dynamic behavior of the electrohydraulic control system for adjustable hydraulic pumps involves the use of concrete values for the physical and geometrical sizes involved in the model. An F316-type axial piston pump with variable dis- placement, made in Romania by UMP, was chosen [10]. The mathematical model was simulated in the MatLab Simulink programming environment [11]. The PID controller was tuned using the Ziegler-Nichols method [13]: $K_{p}=0.3 ; 1 / T_{i}=10$; $K_{D}=0$.

The behavior of the system was analyzed, e.g. the response to the step command for pressure, flow and power. When adjusting the pressure, the control step represents the input signal corresponding to the variation in load pressure from 0 to 200 bar, (Figure 2). When setting the flow, the control step representing the input signal corresponds to the variation in flow from 0 to $30 \mathrm{l} / \mathrm{min}$, (Figure 3 ). In the version of power adjustment the step control that represents the input signal corresponds to a change in power from 0 to $5 \mathrm{~kW}$, (Figure 4).

Simulations indicated that the system can be controlled with the same controller values for any particularly control type.

The dynamic behavior of the system is strongly influenced by the proportionality constant, $K_{p}$, so that the natural frequency of the system increases with this constant, and the system is damped. The integral constant, $T_{i}$, improves the behavior of the steady state, canceling the stationary error. When the value of this constant is increased, it is observed that the damping of the system decreases significantly, bringing it to the limit of instability.

Having a derivative gain, $K_{d}$, even at low levels, the damping of the system decreases, bringing it to the limit of instability.

\section{Experimental research}

In order to achieve the objectives of our study, a stand was designed and constructed that was capable of testing the performance of adjustable pumps working under pressure control, flow, power, or a combination of these features. The overall picture and a schematic representation of the experimental plant is presented in Figure 5. 

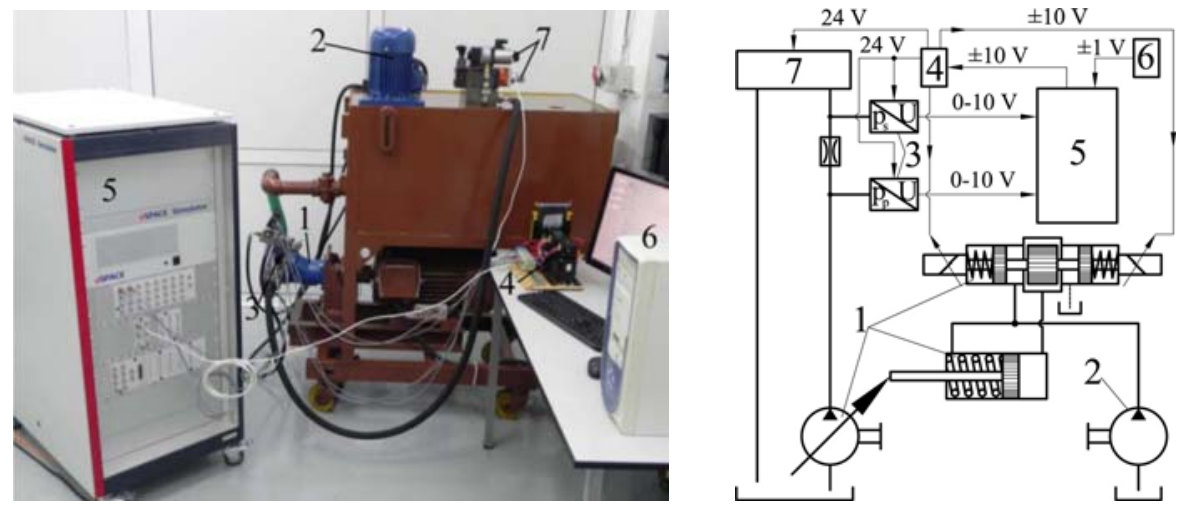

Figure 5: The stand

The main sub-assemblies of the plant are: 1 . the pump, with an electro-hydraulic adjustment system; 2 [8]. the hydraulic energy source for control supply; 3 . a specific sensor; $4[5,12]$. the electricity supply and control system for the proportional distributor, the sensors and the distributor to simulate the load; 5 [4]. the acquisition and control hardware system; 6 [9]. The command and control interface; 7 . the load simulator.

After tuning the controller, the following constants were determined that offer good dynamic behavior for the system regardless of the chosen con- trol type: $K_{p}=0.35 ; 1 / T_{i}=12 ; K_{D}=0$, values very close to those obtained in the simulations, which validates the mathematical model. An important objective of the research was to study the behavior of the PID controller with various control structures. The dynamic behavior of the control structure was investigated by recording the signal step response, which gives the command for pressure, flow and power.

Figures 6 to 8 present in graphical form the data for the set of tests carried out on the stand for the three major types of control.

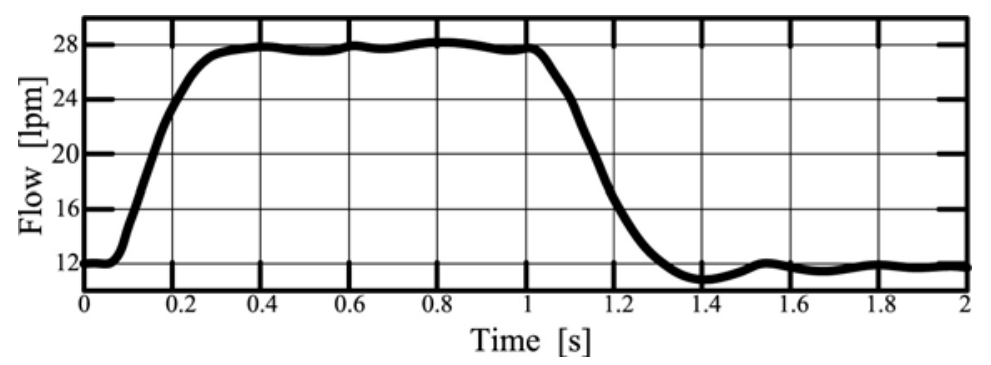

Figure 6: System response to a pressure command of 80 bar, and 20 bar

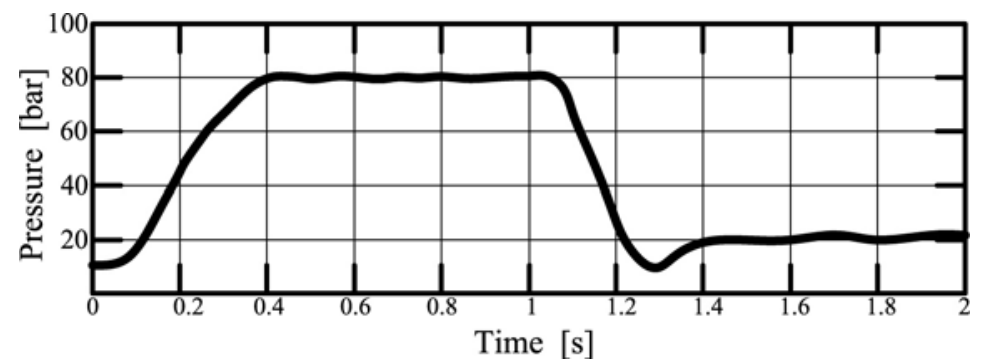

Figure 7: System response to a flow command of 28 bar and $12 \mathrm{lpm}$

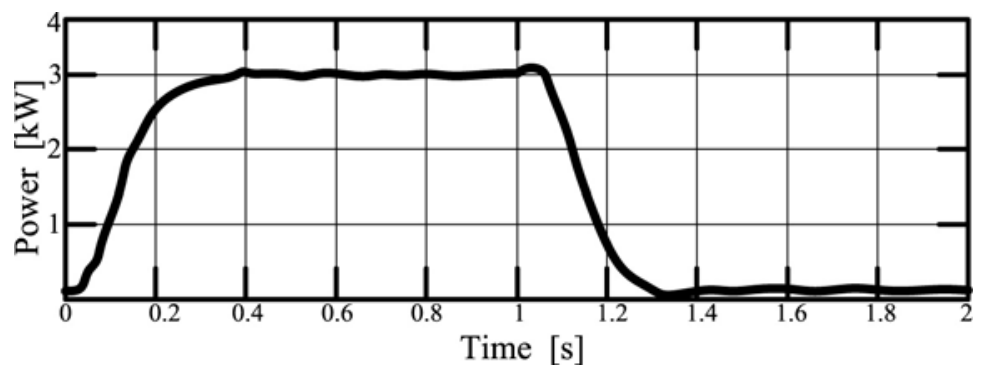

Figure 8: System response to a power command of $3 \mathrm{~kW}$ and $0.1 \mathrm{~kW}$ 
The dynamical behavior of the system is evaluated from the time diagrams (simulated and real diagrams), taking into account the following:

- Stability: The model and the real system are well damped.

- Rapidity: Response Time: 0.2 seconds for the model and 0.4 seconds for the real system, difference is caused by the uncertainty factors that are approximated in the mathematical model.

- Precision: Stationary error below $2 \%$.

The control system has satisfactory behavioral skills for any adjustment structure.

\section{Conclusions}

An analysis of the results indicates that that there are no significant differences between the mathematical model and the real system, so the model can be used for developing new hydraulic machines equipped with this kind of command and control system, which offers the following advantages: Reduced complexity for the circuit construction of the control; it does not involve the use of special expensive equipment; the mechanical structure of the pump is not affected when another hydraulic parameter needs to be controlled; the control scheme is integrated with the piloting and tracking mechanism into a compact smallsize system.

It has been shown that, unlike when using conventional techniques, the three important hydraulic parameters can be adjusted with the same pump, the same controller and only two pressure sensors by simple electrical switching.

Axial piston machines with variable displacement and an electro-hydraulic control system allow complete automation, compatible with the operating cyclograms of complex equipment, by interfacing with a PLC or a process computer.

\section{References}

[1] Banyai, D., Vaida, L.: Electro-hydraulic control system for variable displacement machines, 12th
International Conference on Automation in Production Planning and Manufacturing, p. 50-58, Zilina, Slovakia, 2011.

[2] Banyai, D.: New methods in synthesis of hydraulic machines with variable displacement and electro-hydraulic adjustment, $\mathrm{PhD}$ Thesis, Technical University of Cluj-Napoca, Romania, 2011.

[3] He, Q. H., Hao, P., Zhang, D., Zhang, H. T.: Modeling and Control of Hydraulic Excavator's Arm, Journal of Central South University of Technology, Vol. 13, No. 4, 2006, p. 422-427.

[4] Năşcuţiu, L., Banyai, D., Marcu, I. L: Control system for hydraulic transmissions specific to wind machines, DTMM 2010, Buletinul Institutului Politehnic din Iaşi, Iaşi, Romania, 2010. ISSN 1244-7863.

[5] Năşcuţiu, L., Banyai, D., Opruţa, D.: Measurement of hydraulic parameters, DTMM 2010, Buletinul Institutului Politehnic din Iaşi, Iaşi, Romania, 2010. ISSN 1244-7863.

[6] Opruta, D., Vaida, L.: Dinamica Fluidelor, Ed. Mediamira, Cluj-Napoca, Romania, 2004. ISBN 973-713-044-8.

[7] Vaida, L.: Proportional control for adjustable pumps. PhD Thesis, Technical University of Cluj-Napoca, Romania, 1999.

[8] http://www.boschrexroth.com

[9] http://www.dspace.de

[10] http://www.hidraulica-ph.ro

[11] http://www.mathworks.com

[12] http://www.wika.com

[13] The Control Handbook, CRC Press Catalog Number 8570, 1996. ISBN 0-8493-8375. 